\title{
The Role of Mitochondrial Functional Proteins in ROS Production in Ischemic Heart Diseases
}

\author{
Haifeng Pei, ${ }^{1,2}$ Yi Yang, ${ }^{1}$ Heng Zhao, ${ }^{3}$ Xiuchuan Li, ${ }^{1}$ Dachun Yang, \\ De $\mathrm{Li}^{1}{ }^{1}$ and Yongjian Yang ${ }^{1,2}$ \\ ${ }^{1}$ Department of Cardiology, Chengdu Military General Hospital, Chengdu 610083, China \\ ${ }^{2}$ Department of Cardiology, Third Military Medical University, Chongqing 400042, China \\ ${ }^{3}$ Department of Ultrasonography, Chengdu Military General Hospital, Chengdu 610083, China
}

Correspondence should be addressed to Yongjian Yang; yyj10001@126.com

Received 23 December 2015; Revised 27 January 2016; Accepted 28 January 2016

Academic Editor: Sergio Di Meo

Copyright (C) 2016 Haifeng Pei et al. This is an open access article distributed under the Creative Commons Attribution License, which permits unrestricted use, distribution, and reproduction in any medium, provided the original work is properly cited.

Ischemic heart diseases (IHD) have become the leading cause of death around the world, killing more than 7 million people annually. In IHD, the blockage of coronary vessels will cause irreversible cell injury and even death. As the "powerhouse" and "apoptosis center" in cardiomyocytes, mitochondria play critical roles in IHD. Ischemia insult can reduce myocardial ATP content, resulting in energy stress and overproduction of reactive oxygen species (ROS). Thus, mitochondrial abnormality has been identified as a hallmark of multiple cardiovascular disorders. To date, many studies have suggested that these mitochondrial proteins, such as electron transport chain (ETC) complexes, uncoupling proteins (UCPs), mitochondrial dynamic proteins, translocases of outer membrane (Tom) complex, and mitochondrial permeability transition pore (MPTP), can directly or indirectly influence mitochondria-originated ROS production, consequently determining the degree of mitochondrial dysfunction and myocardial impairment. Here, the focus of this review is to summarize the present understanding of the relationship between some mitochondrial functional proteins and ROS production in IHD.

\section{Introduction}

As the leading cause of fatality worldwide, ischemic heart diseases (IHD) give rise to widespread loss of cardiomyocytes and subsequent adverse cardiac remodeling [1]. More than 17 million people had succumbed to IHD in 2008, and the number was estimated to be 23.6 million by 2030 [2]. Ischemia starves cardiomyocytes of vital oxygen, resulting in severe or irreversible injury to heart [3]. Abundant lines of evidence indicate that reactive oxygen species (ROS) overload that originates from mitochondria is closely linked with the pathogenesis of cardiovascular diseases, such as atherosclerosis, myocardial infarction, and heart failure [4]. In myocytes, mitochondria comprise more than $30 \%$ cell volume and generate about $90 \%$ of the ATP [5]. Accordingly, mitochondria are also the major source of ROS in the cardiovascular diseases $[5,6]$. Numerous experimental and clinical studies have shown that ROS accumulation is significantly exacerbated in the failing myocardium [7]. Chronic exposure of myocytes to ROS will lead to apoptosis, necrosis, and fibrosis, finally causing arrhythmias, impairment of excitationcontraction coupling, and cardiac remodeling [8-10]. Given that ROS affect virtually all aspects of cardiac myocyte physiology and that those lines of evidence support the notion that ROS are increased in IHD, the underlying mechanisms about ROS overproduction induced by mitochondrial abnormality should be further explored.

\section{The Electron Transport Chain (ETC) Complexes and Uncoupling Proteins (UCPs)}

Mitochondrial oxidative phosphorylation is the basic mechanism of ATP synthesis, in which the five enzyme complexes $\mathrm{I}-\mathrm{V}$ in mitochondria are composed of multiple subunits. The respiratory chain, apart from energy production, plays a pivotal role in regulation of cardiac oxidative stress [11]. 
ETC damage will lead to a robust increase of ROS production in mitochondria [12]. In ischemic heart, oxygen delivery to myocardium is not sufficient to meet the demand of mitochondrial oxidation. Reintroduction of oxygen induced by reperfusion markedly enhances electron leakage from the ETC, resulting in overproduction of superoxide $\left(\mathrm{O}_{2}{ }^{-}\right)$and $\mathrm{O}_{2}{ }^{-}$-derived oxidants $[12,13]$. Indeed, reduced activity of ETC subunits in heart failure patients has been confirmed independently of the etiology, notably of complex I [14], complex III [15], and complex IV [16]. Within the ETC, complexes I/III are regarded as the key contributors to ROS generation under stressful conditions $[17,18]$. In the setting of myocardial ischemia/reperfusion (MI/R), oxidative injury of complex I can weaken complex interaction and enhance ROS production by complex III $[19,20]$. Although the contribution of intact complex II seems to be negligible, some mutations in subunits of complex II have been linked to specific forms of cancer or neurodegeneration with increased ROS generation [21, 22]. Aldakkak et al. [23] revealed that pharmacologic blockade of electron transport attenuates ROS-induced cardiac injury. Consistently, nitrite can modulate mitochondrial resilience to reperfusion injury, perhaps via inhibiting complex I by posttranslational s-nitrosation to reduce ROS generation $[24,25]$. Moreover, ROS scavenging ability may be impaired as an evident decrease of MnSOD activity in failing heart [26]. Coenzyme Q (CoQ) encompasses a collection of homologous molecules which consist of a benzoquinone ring linked to an isoprenoid side chain [27]. In the quinone form, CoQ serves as an electron carrier to transfer electrons in the mitochondrial ETC. In the quinol form (the reduced form of quinone), CoQ represents an efficient antioxidant in the body [28]. CoQ10 at high doses has also been proved to regulate gene transcription, including the genes important in lipid metabolism and the specific genes induced by ROS-sensitive intracellular pathways [29]. It is claimed that CoQ10 supplementation to obese rats attenuates age-related oxidative stress and preserves mitochondrial function in myocardium [30]. Moreover, CoQ10 deficiency is detrimental to the prognosis of chronic heart failure, and plasma concentration of CoQ10 may serve as an independent predictor of mortality [31]. Long-term supplementation of CoQ10 for patients with heart failure improves symptoms and reduces major adverse cardiovascular events [32].

In contrast, uncoupling proteins (UCPs) that are located in mitochondrial inner membrane exhibit opposite functions as complexes mentioned above. UCPs can reduce mitochondrial ROS production by dissipating the electrochemical gradient [33-36]. UCPs overexpression was found to salvage cardiomyocytes by preserving mitochondrial integrity $[33,36]$. Specifically, mild to moderate mitochondrial uncoupling mediated by UCPs protects against MI/R injury by reducing ROS generation [37]. UCP1, characterized in brown adipocytes [38], has close relationship with endogenous CoQ to influence $\mathrm{H}^{+}$transport [39]. In the setting of hypoxia/reoxygenation, UCP1 overexpression confers cardioprotection [33]. The presence of UCP1 mitigates reperfusion-induced damage, probably by reducing mitochondrial hyperpolarization [40]. Moreover, UCP2 overexpression can exert cardioprotection perhaps by preventing mitochondrial $\mathrm{Ca}^{2+}$ overload and attenuating ROS generation [41]. UCP3 has also been reported to play a critical role in cardioprotection during oxidative stress by suppressing detrimental ROS generation and maintaining myocardial high-energy phosphates [37]. In view of what was mentioned above, UCPs may be developed as protective targets for IHD patients.

\section{Mitochondrial Dynamic Proteins}

In order to adapt to stressful conditions, mitochondria dynamically undergo fission and fusion [42], which is basically regulated by some functional proteins [42, 43]. Mitofusin 1/2 (Mfn1/2) and optic atrophy 1 (Opal) are known to induce mitochondrial fusion $[44,45]$, while dynaminrelated protein 1 (Drpl) is identified to interact with fission protein 1 (Fis1) to promote mitochondrial fission [46, 47]. In mammalian cells, mitochondrial function is largely governed by mitochondrial fission and fusion which is an important factor for the integrity, structure, and function of healthy mitochondria. Abnormal fission or fusion will impair mitochondrial function, for example, resulting in overload of mitochondrial $\mathrm{Ca}^{2+}$, overproduction of free radicals, alteration of mitochondrial enzymatic activities, and reduction of ATP production [48].

Recent studies have highlighted the notion that defects in mitochondrial dynamics are relevant to various human disorders, including MI/R, heart failure, diabetes, and aging $[49,50]$. Inhibition of mitochondrial fission has been considered to rescue myocardial infarction/heart failure [7]. The deletion of Mfn1/2 appears to render the heart less resistant to MPTP opening [51], whereas overexpression of Mfn1/2 prevents the opening of MPTP and reduces cell death following ischemia/reperfusion (I/R) [52, 53]. Opal mutation in myocardium results in mitochondrial dysfunction and increases ROS [54], while transfection of Opal in vivo protects mice from denervation-induced ischemic heart damage [55]. Moreover, inhibition of Drpl significantly decreased I/R-induced mitochondrial fragmentation and cardiomyocytes apoptosis $[52,56]$. Cumulative lines of evidence suggest that impaired mitochondrial dynamics are an early event in the progression of IHD that involve excessive mitochondrial fission and mitochondrial dysfunction, indicating that dynamics related proteins may be developed as new therapeutic targets against IHD.

\section{The Translocases of Outer Membrane (Tom) Complex}

The translocases of mitochondrial outer membrane (Tom) complex is a general entry gate for the importing of all mitochondrial preproteins. It comprises a central channel (formed by Tom40, Tom5, Tom6, and Tom7) and three receptors (Tom70, Tom20, and Tom22) [57]. Tom 20 and Tom70 can recognize mitochondrial-targeting sequences on the precursor proteins and transfer them to Tom 22 and the central channel $[57,58]$. Tom 20 and Tom 22 mainly recognize the presequences of cleavable precursor proteins, while 
Tom70 typically largely recognizes the internal targeting signals of hydrophobic precursor proteins, many of which are inner membrane metabolite carriers [58]. In addition, Tom70 can also function as a docking site for cytosolic chaperones, such as Hsp70 and Hsp90, in order to receive mitochondrial proteins [59].

It has been reported that mitochondrial Tom 20 was reduced by ischemia, and the maintenance of Tom 20 by preconditioning confers cardioprotection via improving mitochondrial structure and function [60]. Tom20 can also restore the translocation of $\mathrm{Cx} 43$ to the inner mitochondrial membrane to attenuate ischemic myocardial injury [60]. Conversely, proteasome inhibitors, such as MG115, proteasome inhibitor 1, and lactacystin, lead to higher levels of Tom20 and enhance the perinuclear accumulation of mitochondria, revealing the influence of cellular redox conditions on mitochondrial import [61]. Moreover, Tom70 is obviously suppressed in hypertrophic hearts, and genetic downregulation of Tom70 worsens pathological cardiomyocyte hypertrophy. The defective mitochondrial import of Tom70-targeted Opal triggered intracellular oxidative stress, which led to a pathological cellular response [62]. Adenosine administration in ischemic myocardium may exhibit protection by inducing the translocation of PKCE to mitochondria in a Tom70/HSP90dependent manner [63]. However, the detailed mechanisms about Tom complex in IHD remain obscured to date, so further studies are needed.

\section{The Other Functional Proteins in Mitochondrion}

5.1. Mitochondrial Permeability Transition Pore (MPTP). MPTP is a nonselective channel locating on the inner mitochondrial membrane. ROS overproduction and calcium overload can dissipate the proton electrochemical gradient and open the MPTP, leading to uncoupling of oxidative phosphorylation and further production of ROS [64, 65]. The opening of MPTP will release proapoptotic proteins to induce cell apoptosis or necrosis $[13,65]$. Several studies have demonstrated that MPTP inhibition can mitigate cell loss in cardiac pathologies, including MI/R [66], heart failure [67], and diabetic cardiomyopathy [68]. Hausenloy and his colleagues revealed that inhibition of MPTP opening, during the first few minutes of reperfusion, protects myocytes from oxidative stress and finally limits infarct size [69]. However, Saotome et al. claimed that ROS-induced transient opening of MPTP protects from I/R-induced myocardium injury [70], revealing that the specific function of MPTP depends on the degree and the timing.

5.2. Mitochondrial $\mathrm{Ca}^{2+}$ Uniporter (MCU)/Mitochondrial $\mathrm{Ca}^{2+}$ Uptake 1 (MICU1)/MICU2. As a universal secondary messenger, $\mathrm{Ca}^{2+}$ plays a central role in a wide range of cellular processes, such as muscular contraction, synaptic transmission, cell migration, and cell proliferation [71]. In the heart, $\mathrm{Ca}^{2+}$ is central to cardiac excitation-contraction coupling and signaling networks in the regulation of pathological myocardial growth and remodeling [72]. Accumulating lines of evidence indicate that $\mathrm{Ca}^{2+}$ overload is linked with mitochondrial dysfunction, contractile dysfunction, and cell death $[23,73-77]$. Thus, maintenance of mitochondrial $\mathrm{Ca}^{2+}$ homeostasis is very important for the survival of cardiomyocytes under ischemic stress [78]. MCU is an inner mitochondrial membrane channel responsible for $\mathrm{Ca}^{2+}$ uptake into the matrix [79]. It plays a fundamental role in the control of aerobic metabolism as well as apoptosis [80]. MCU blockade may protect the heart from hypoxia/reoxygenation injury through suppressing mitochondria-originated production of ROS [81]. Recently, MICU1 and MICU2 are identified to function as the gatekeepers of MCU by the binding of $\mathrm{Ca}^{2+}$ to their $\mathrm{EF}$ hands [82]. And MICU2's activity requires the presence of MICU1 [83]. Under basal cytosolic $\left[\mathrm{Ca}^{2+}\right]$ condition, $\mathrm{Ca}^{2+}$. free MICU1 and MICU2 can inhibit MCU function; at very high cytosolic $\left[\mathrm{Ca}^{2+}\right]$ condition, all EF hands of MICU1 and MICU2 bind $\mathrm{Ca}^{2+}$ to activate MCU by dissociation of MICUs from MCU complex [82]. Consistently, Mallilankaraman et al. [84] prove that MICU1 is required to preserve mitochondrial $\left[\mathrm{Ca}^{2+}\right]$, under basal condition. In the absence of MICU1, mitochondria suffers $\mathrm{Ca}^{2+}$ overload, leading to excessive ROS generation. In addition, Patron et al. [85] also reveal that MICU2 largely shuts down MCU activity at low cytosolic $\left[\mathrm{Ca}^{2+}\right]$, whereas in response to high cytosolic $\left[\mathrm{Ca}^{2+}\right]$, MICU1 appears to stimulate $\mathrm{Ca}^{2+}$ uptake by MCU. Very recently, Wang et al. claim that Mfn2 overexpression reduces the expression of MICU1 and MICU2 to trigger influx of $\mathrm{Ca}^{2+}$ into mitochondria [86], revealing the close relationship of mitochondrial dynamics with $\mathrm{Ca}^{2+}$ homeostasis. However, the full functions of MICU1 and MICU2 in cardiovascular diseases remain unclear yet, emphasizing an urgent need for deep research.

5.3. Connexin 43 (Cx43). Gap junction channels provide the basis for intercellular communication in cardiovascular systems, in order to keep metabolic interchange and maintain normal cardiac rhythm. In cardiomyocytes, $\mathrm{Cx} 43$ is the most abundant isoform. The alteration of $\mathrm{Cx} 43$ was observed in myocardium diseases, such as ischemia, hypertrophic cardiomyopathy, and heart failure [87]. The normal location of $\mathrm{Cx} 43$ at inner mitochondrial membrane is very important in the synchronization of contraction for cardiomyocytes $[88,89]$. It was suggested that $I / R$ injury is accompanied with the change of $\mathrm{Cx} 43$ expression [90]. Ischemic conditions can trigger Cx43 hemichannel opening, possibly mediated by the generation of ROS and nitrogen species [91]. The protection of preconditioning has been confirmed to depend on functional Cx43-formed channels [2]. Moreover, Cx43 deficiency will lead to ventricular arrhythmia which is the major cause of sudden death in heart failure. The proteasome inhibitor can be used to attenuate the degradation of $\mathrm{Cx} 43$ to prevent $\mathrm{Cx} 43$-mediated arrhythmia in heart failure [92]. Therefore, $\mathrm{Cx} 43$ may be developed as a potential therapeutic target for cardiovascular diseases [90].

5.4. Signal Transducer and Activator of Transcription 3 (STAT3). As a transcription factor, STAT3 has been implicated to protect hearts from acute ischemic injury under 


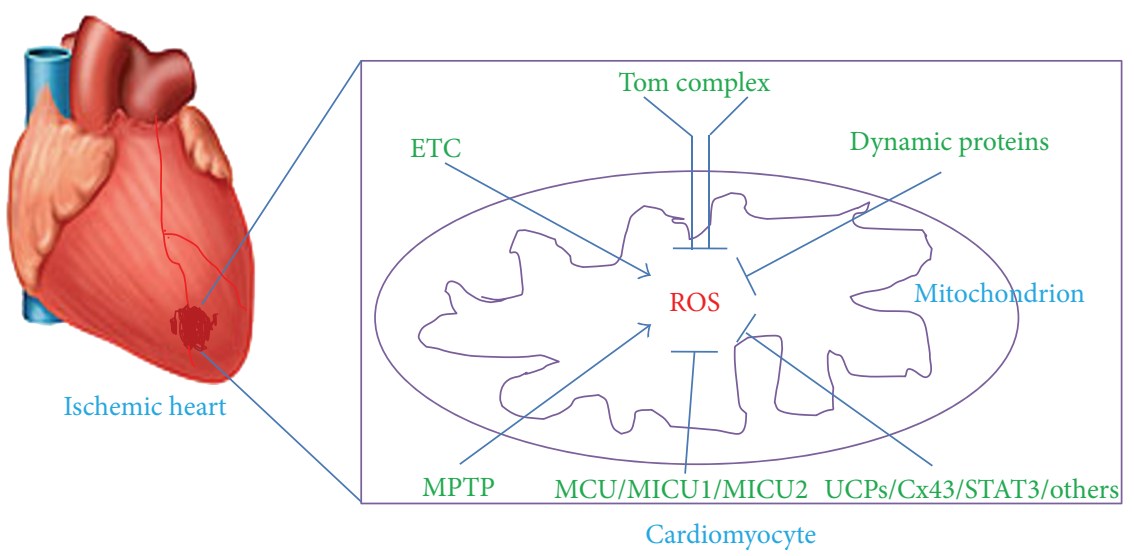

FIGURE 1: Schematic diagram indicating mitochondrial proteins' role in ROS generation in ischemic heart diseases. Under ischemic insult, the generation of ROS significantly increases in mitochondria of cardiomyocytes, which can be directly or indirectly influenced by mitochondrial functional proteins, including ETC complexes, UCPs, mitochondrial dynamic proteins, Tom complex, MPTP, MCU/MICU1/MICU2, Cx43, and STAT3. ROS: reactive oxygen species; ETC: electron transport chain; UCPs: uncoupling proteins; Tom: translocases of outer membrane;

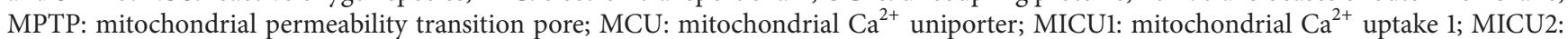
mitochondrial $\mathrm{Ca}^{2+}$ uptake 2; Cx43: Connexin 43; STAT3: signal transducer and activator of transcription 3.

stressful conditions [93], and loss of STAT3 will result in cardiomyopathy [94]. Accumulative lines of evidence also demonstrated that STAT3 serves as a protective molecule for the heart in hypertension and advanced age [95]. STAT3 is now known to be present in cardiac mitochondria and be able to regulate ROS generation and MPTP opening [96]. Wegrzyn and colleagues have proved that STAT3 deficiency can reduce the activities of complexes I and II [97]. Szczepanek et al. further revealed that mutation in the DNA binding domain of mitochondrial-targeted STAT3 can disrupt ETC activity in the heart [96]. Consistently, STAT3 transgene reduces the vulnerability of cardiac mitochondria to ischemia by restoring complex I activity and suppressing ROS generation [98]. Thus, in pathologic settings such as ischemia or early reperfusion, STAT3 may be an ideal therapeutic target to protect cardiac mitochondria.

\section{Conclusion}

In summary, mitochondrial functional proteins play critical roles in the production of ROS in IHD: (1) the defective ETC activity, notably of decreased activity of complex I, may form the pathological foundation for mitochondriaderived ROS overload; (2) the disruption of mitochondrial dynamics, especially depressed mitochondrial fusion, will aggravate mitochondrial ROS production; (3) Tom complex may possess important property in regulating oxidative stress, perhaps via influencing the translocation of mitochondrial proteins; (4) the other functional factors, such as MPTP, MCU/MICU1/MICU2, Cx43, and STAT3, play important roles in preserving mitochondrial integrity and function, directly or indirectly through inhibiting ROS overload (Figure 1).

In recent decades, there has been great progress in screening, identifying, and developing molecules as therapeutic targets to preserve mitochondrial integrity and prevent ROS overload. As we understand more of the distinct abnormalities occurring in the mitochondria with IHD, the goal becomes to develop new methods to mitigate the mitochondrial abnormalities. Current medicines, such beta blockers, statins, and nitrates, have improved the symptoms of IHD patients; however, these medicines' effects on mitochondrial impairment and mitochondrial functional proteins in IHD are far from clear yet. Undoubtedly, more work is needed to explore the fundamental roles of mitochondrial proteins in IHD, because they are attractive mechanistic targets for cardioprotection.

\section{Conflict of Interests}

The authors declare that they have no conflict of interests.

\section{Authors' Contribution}

Haifeng Pei and Yi Yang contributed equally to this study.

\section{Acknowledgments}

This work was supported by grants from the National Science Funds of China (nos. 81500208 and 81470396), Youth Breeding Project for Medical Scientific Research Program of PLA (no. 14QNP050), and Science \& Technology Project of Sichuan Province (no. 2015JY0277).

\section{References}

[1] V. L. Roger, A. S. Go, D. M. Lloyd-Jones et al., "Executive summary: heart disease and stroke statistics-2012 update: a report from the American Heart Association," Circulation, vol. 125, no. 1, pp. 188-197, 2012.

[2] H. He, N. Li, Z. Zhao, F. Han, X. Wang, and Y. Zeng, "Ischemic postconditioning improves the expression of cellular membrane connexin 43 and attenuates the reperfusion injury in rat acute 
myocardial infarction," Biomedical Reports, vol. 3, pp. 668-674, 2015.

[3] W. H. Townley-Tilson, X. Pi, and L. Xie, "The role of oxygen sensors, hydroxylases, and hif in cardiac function and disease," Oxidative Medicine and Cellular Longevity, vol. 2015, Article ID 676893, 10 pages, 2015.

[4] Z. W. Zhang, X. C. Xu, T. Liu, and S. Yuan, "Mitochondrionpermeable antioxidants to treat ROS-burst-mediated acute diseases," Oxidative Medicine and Cellular Longevity, vol. 2016, Article ID 6859523, 10 pages, 2016.

[5] E. Murphy and C. Steenbergen, "Preconditioning: the mitochondrial connection," Annual Review of Physiology, vol. 69, pp. 51-67, 2007.

[6] D. B. Zorov, C. R. Filburn, L.-O. Klotz, J. L. Zweier, and S. J. Sollott, "Reactive oxygen species (ROS)-induced ROS release: a new phenomenon accompanying induction of the mitochondrial permeability transition in cardiac myocytes," The Journal of Experimental Medicine, vol. 192, no. 7, pp. 1001-1014, 2000.

[7] A. A. Knowlton, L. Chen, and Z. A. Malik, "Heart failure and mitochondrial dysfunction: the role of mitochondrial fission/fusion abnormalities and new therapeutic strategies," Journal of Cardiovascular Pharmacology, vol. 63, no. 3, pp. 196206, 2014.

[8] F. J. Giordano, "Oxygen, oxidative stress, hypoxia, and heart failure," The Journal of Clinical Investigation, vol. 115, no. 3, pp. 500-508, 2005.

[9] C. Maack, E. R. Dabew, M. Hohl, H.-J. Schäfers, and M. Böhm, "Endogenous activation of mitochondrial KATP channels protects human failing myocardium from hydroxyl radicalinduced stunning," Circulation Research, vol. 105, no. 8, pp. 811817, 2009.

[10] F. G. Akar, M. A. Aon, G. F. Tomaselli, and B. O’Rourke, “The mitochondrial origin of postischemic arrhythmias," The Journal of Clinical Investigation, vol. 115, no. 12, pp. 3527-3535, 2005.

[11] K. Schwarz, N. Siddiqi, S. Singh, C. J. Neil, D. K. Dawson, and M. P. Frenneaux, "The breathing heart-mitochondrial respiratory chain dysfunction in cardiac disease," International Journal of Cardiology, vol. 171, no. 2, pp. 134-143, 2014.

[12] T. Ide, H. Tsutsui, S. Kinugawa et al., "Mitochondrial electron transport complex I is a potential source of oxygen free radicals in the failing myocardium," Circulation Research, vol. 85, no. 4, pp. 357-363, 1999.

[13] E. Murphy and C. Steenbergen, "Mechanisms underlying acute protection from cardiac ischemia-reperfusion injury," Physiological Reviews, vol. 88, no. 2, pp. 581-609, 2008.

[14] R. J. Scheubel, M. Tostlebe, A. Simm et al., "Dysfunction of mitochondrial respiratory chain complex I in human failing myocardium is not due to disturbed mitochondrial gene expression," Journal of the American College of Cardiology, vol. 40, no. 12, pp. 2174-2181, 2002.

[15] D. Jarreta, J. Orús, A. Barrientos et al., "Mitochondrial function in heart muscle from patients with idiopathic dilated cardiomyopathy," Cardiovascular Research, vol. 45, no. 4, pp. 860-865, 2000.

[16] E. Arbustini, M. Diegoli, R. Fasani et al., "Mitochondrial DNA mutations and mitochondrial abnormalities in dilated cardiomyopathy," American Journal of Pathology, vol. 153, no. 5, pp. 1501-1510, 1998.

[17] M. P. Murphy, "How mitochondria produce reactive oxygen species," The Biochemical Journal, vol. 417, no. 1, pp. 1-13, 2009.
[18] M. D. Brand, "The sites and topology of mitochondrial superoxide production," Experimental Gerontology, vol. 45, no. 7-8, pp. 466-472, 2010.

[19] Q. Chen, A. K. S. Camara, D. F. Stowe, C. L. Hoppel, and E. J. Lesnefsky, "Modulation of electron transport protects cardiac mitochondria and decreases myocardial injury during ischemia and reperfusion," American Journal of PhysiologyCell Physiology, vol. 292, no. 1, pp. C137-C147, 2007.

[20] Q. Chen, C. L. Hoppel, and E. J. Lesnefsky, "Blockade of electron transport before cardiac ischemia with the reversible inhibitor amobarbital protects rat heart mitochondria," The Journal of Pharmacology and Experimental Therapeutics, vol. 316, no. 1, pp. 200-207, 2006.

[21] V. Yankovskaya, R. Horsefield, S. Törnroth et al., "Architecture of succinate dehydrogenase and reactive oxygen species generation," Science, vol. 299, no. 5607, pp. 700-704, 2003.

[22] T. M. Iverson, E. Maklashinas, and G. Cecchinis, "Structural basis for malfunction in complex II," The Journal of Biological Chemistry, vol. 287, no. 42, pp. 35430-35438, 2012.

[23] M. Aldakkak, D. F. Stowe, Q. Chen, E. J. Lesnefsky, and A. K. S. Camara, "Inhibited mitochondrial respiration by amobarbital during cardiac ischaemia improves redox state and reduces matrix $\mathrm{Ca}^{2+}$ overload and ROS release," Cardiovascular Research, vol. 77, no. 2, pp. 406-415, 2008.

[24] S. Shiva, M. N. Sack, J. J. Greer et al., "Nitrite augments tolerance to ischemia/reperfusion injury via the modulation of mitochondrial electron transfer," The Journal of Experimental Medicine, vol. 204, no. 9, pp. 2089-2102, 2007.

[25] S. M. Nadtochiy, L. S. Burwell, and P. S. Brookes, "Cardioprotection and mitochondrial S-nitrosation: effects of Snitroso-2-mercaptopropionyl glycine (SNO-MPG) in cardiac ischemia-reperfusion injury," Journal of Molecular and Cellular Cardiology, vol. 42, no. 4, pp. 812-825, 2007.

[26] F. Sam, D. L. Kerstetter, D. R. Pimental et al., "Increased reactive oxygen species production and functional alterations in antioxidant enzymes in human failing myocardium," Journal of Cardiac Failure, vol. 11, no. 6, pp. 473-480, 2005.

[27] J. L. Tarry-Adkins, H. L. Blackmore, M. S. Martin-Gronert et al., "Coenzyme Q10 prevents accelerated cardiac aging in a rat model of poor maternal nutrition and accelerated postnatal growth," Molecular Metabolism, vol. 2, no. 4, pp. 480-490, 2013.

[28] M. Bentinger, K. Brismar, and G. Dallner, "The antioxidant role of coenzyme Q," Mitochondrion, vol. 7, supplement, pp. S41-S50, 2007.

[29] C. Schmelzer, M. Kitano, K. Hosoe, and F. Döring, "Ubiquinol affects the expression of genes involved in PPAR $\alpha$ signalling and lipid metabolism without changes in methylation of $\mathrm{CpG}$ promoter islands in the liver of mice," Journal of Clinical Biochemistry and Nutrition, vol. 50, no. 2, pp. 119-126, 2012.

[30] J. J. Ochoa, J. L. Quiles, J. R. Huertas, and J. Mataix, "Coenzyme Q10 protects from aging-related oxidative stress and improves mitochondrial function in heart of rats fed a polyunsaturated fatty acid (PUFA)-rich diet," The Journals of Gerontology Series A: Biological Sciences and Medical Sciences, vol. 60, no. 8, pp. 970-975, 2005.

[31] S. L. Molyneux, C. M. Florkowski, P. M. George et al., "Coenzyme Q10: an independent predictor of mortality in chronic heart failure," Journal of the American College of Cardiology, vol. 52, no. 18, pp. 1435-1441, 2008.

[32] S. A. Mortensen, F. Rosenfeldt, A. Kumar et al., "The effect of coenzyme $\mathrm{Q}_{10}$ on morbidity and mortality in chronic heart 
failure: results from Q-SYMBIO: a randomized double-blind trial," JACC: Heart Failure, vol. 2, no. 6, pp. 641-649, 2014.

[33] M. Bienengraeber, C. Ozcan, and A. Terzic, "Stable transfection of UCP1 confers resistance to hypoxia/reoxygenation in a heartderived cell line," Journal of Molecular and Cellular Cardiology, vol. 35, no. 7, pp. 861-865, 2003.

[34] C. J. McLeod, A. Aziz, R. F. Hoyt Jr., J. P. McCoy Jr., and M. N. Sack, "Uncoupling proteins 2 and 3 function in concert to augment tolerance to cardiac ischemia," The Journal of Biological Chemistry, vol. 280, no. 39, pp. 33470-33476, 2005.

[35] S. M. Nadtochiy, A. J. Tompkins, and P. S. Brookes, "Different mechanisms of mitochondrial proton leak in ischaemia/reperfusion injury and preconditioning: implications for pathology and cardioprotection," The Biochemical Journal, vol. 395, no. 3, pp. 611-618, 2006.

[36] M. N. Sack, "Mitochondrial depolarization and the role of uncoupling proteins in ischemia tolerance," Cardiovascular Research, vol. 72, no. 2, pp. 210-219, 2006.

[37] C. Ozcan, M. Palmeri, T. L. Horvath, K. S. Russell, and R. R. Russell III, "Role of uncoupling protein 3 in ischemiareperfusion injury, arrhythmias, and preconditioning," The American Journal of Physiology-Heart and Circulatory Physiology, vol. 304, no. 9, pp. H1192-H1200, 2013.

[38] S. Krauss, C.-Y. Zhang, and B. B. Lowell, "The mitochondrial uncoupling-protein homologues," Nature Reviews Molecular Cell Biology, vol. 6, no. 3, pp. 248-261, 2005.

[39] K. S. Echtay, E. Winkler, and M. Klingenberg, "Coenzyme Q is an obligatory cofactor for uncoupling protein function," Nature, vol. 408, no. 6812, pp. 609-613, 2000.

[40] J. Hoerter, M.-D. Gonzalez-Barroso, E. Couplan et al., "Mitochondrial uncoupling protein 1 expressed in the heart of transgenic mice protects against ischemic-reperfusion damage," Circulation, vol. 110, no. 5, pp. 528-533, 2004.

[41] Y. Teshima, M. Akao, S. P. Jones, and E. Marbán, “Uncoupling protein-2 overexpression inhibits mitochondrial death pathway in cardiomyocytes," Circulation Research, vol. 93, no. 3, pp. 192200, 2003.

[42] R. J. Youle and A. M. van der Bliek, "Mitochondrial fission, fusion, and stress," Science, vol. 337, no. 6098, pp. 1062-1065, 2012.

[43] M. Liesa, M. Palacín, and A. Zorzano, "Mitochondrial dynamics in mammalian health and disease," Physiological Reviews, vol. 89, no. 3, pp. 799-845, 2009.

[44] T. Koshiba, S. A. Detmer, J. T. Kaiser, H. Chen, J. M. McCaffery, and D. C. Chan, "Structural basis of mitochondrial tethering by mitofusin complexes," Science, vol. 305, no. 5685, pp. 858-862, 2004.

[45] S. Cipolat, O. Martins de Brito, B. Dal Zilio, and L. Scorrano, "OPA1 requires mitofusin 1 to promote mitochondrial fusion," Proceedings of the National Academy of Sciences of the United States of America, vol. 101, no. 45, pp. 15927-15932, 2004.

[46] E. Smirnova, L. Griparic, D.-L. Shurland, and A. M. van der Bliek, "Dynamin-related protein Drpl is required for mitochondrial division in mammalian cells," Molecular Biology of the Cell, vol. 12, no. 8, pp. 2245-2256, 2001.

[47] Y. Yoon, E. W. Krueger, B. J. Oswald, and M. A. McNiven, "The mitochondrial protein hFis1 regulates mitochondrial fission in mammalian cells through an interaction with the dynamin-like protein DLP1," Molecular and Cellular Biology, vol. 23, no. 15, pp. 5409-5420, 2003.
[48] P. H. Reddy, "Inhibitors of mitochondrial fission as a therapeutic strategy for diseases with oxidative stress and mitochondrial dysfunction," Journal of Alzheimer's Disease, vol. 40, no. 2, pp. 245-256, 2014.

[49] W. W. Sharp, Y. H. Fang, M. Han et al., "Dynamin-related protein 1 (Drp1)-mediated diastolic dysfunction in myocardial ischemia-reperfusion injury: therapeutic benefits of Drpl inhibition to reduce mitochondrial fission," The FASEB Journal, vol. 28, no. 1, pp. 316-326, 2014.

[50] J. Marín-García, A. T. Akhmedov, and G. W. Moe, "Mitochondria in heart failure: the emerging role of mitochondrial dynamics," Heart Failure Reviews, vol. 18, no. 4, pp. 439-456, 2013.

[51] L. Chen, Q. Gong, J. P. Stice, and A. A. Knowlton, "Mitochondrial OPA1, apoptosis, and heart failure," Cardiovascular Research, vol. 84, no. 1, pp. 91-99, 2009.

[52] S.-B. Ong, S. Subrayan, S. Y. Lim, D. M. Yellon, S. M. Davidson, and D. J. Hausenloy, "Inhibiting mitochondrial fission protects the heart against ischemia/reperfusion injury," Circulation, vol. 121, no. 18, pp. 2012-2022, 2010.

[53] K. N. Papanicolaou, R. J. Khairallah, G. A. Ngoh et al., "Mitofusin-2 maintains mitochondrial structure and contributes to stress-induced permeability transition in cardiac myocytes," Molecular and Cellular Biology, vol. 31, no. 6, pp. 1309-1328, 2011.

[54] L. Chen, T. Liu, A. Tran et al., "OPA1 mutation and lateonset cardiomyopathy: mitochondrial dysfunction and mtDNA instability," Journal of the American Heart Association, vol. 1, Article ID e003012, 2012.

[55] T. Varanita, M. E. Soriano, V. Romanello et al., "The OPA1dependent mitochondrial cristae remodeling pathway controls atrophic, apoptotic, and ischemic tissue damage," Cell Metabolism, vol. 21, no. 6, pp. 834-844, 2015.

[56] M. Sumida, K. Doi, E. Ogasawara et al., "Regulation of mitochondrial dynamics by dynamin-related protein-1 in acute cardiorenal syndrome," Journal of the American Society of Nephrology, vol. 26, no. 10, pp. 2378-2387, 2015.

[57] A. Chacinska, C. M. Koehler, D. Milenkovic, T. Lithgow, and N. Pfanner, "Importing mitochondrial proteins: machineries and mechanisms," Cell, vol. 138, no. 4, pp. 628-644, 2009.

[58] O. Schmidt, N. Pfanner, and C. Meisinger, "Mitochondrial protein import: from proteomics to functional mechanisms," Nature Reviews Molecular Cell Biology, vol. 11, no. 9, pp. 655667, 2010.

[59] J. C. Young, N. J. Hoogenraad, and F. U. Hartl, "Molecular chaperones Hsp90 and Hsp70 deliver preproteins to the mitochondrial import receptor Tom70," Cell, vol. 112, no. 1, pp. 41-50, 2003.

[60] K. Boengler, P. Gres, A. Cabestrero et al., "Prevention of the ischemia-induced decrease in mitochondrial Tom 20 content by ischemic preconditioning," Journal of Molecular and Cellular Cardiology, vol. 41, no. 3, pp. 426-430, 2006.

[61] G. Wright, K. Terada, M. Yan, I. Sergeev, and M. Mori, “Oxidative stress inhibits the mitochondrial import of preproteins and leads to their degradation," Experimental Cell Research, vol. 263, no. 1, pp. 107-117, 2001.

[62] J. Li, M. Qi, C. Li et al., "Tom70 serves as a molecular switch to determine pathological cardiac hypertrophy," Cell Research, vol. 24, no. 8, pp. 977-993, 2014. 
[63] Z. Yang, W. Sun, and K. Hu, "Molecular mechanism underlying adenosine receptor-mediated mitochondrial targeting of protein kinase C," Biochimica et Biophysica Acta (BBA) - Molecular Cell Research, vol. 1823, no. 4, pp. 950-958, 2012.

[64] D. J. Hausenloy and D. M. Yellon, “The mitochondrial permeability transition pore: Its fundamental role in mediating cell death during ischaemia and reperfusion," Journal of Molecular and Cellular Cardiology, vol. 35, no. 4, pp. 339-341, 2003.

[65] C. P. Baines, "The molecular composition of the mitochondrial permeability transition pore," Journal of Molecular and Cellular Cardiology, vol. 46, no. 6, pp. 850-857, 2009.

[66] J. Zhu, M. J. Rebecchi, P. S. A. Glass, P. R. Brink, and L. Liu, "Cardioprotection of the aged rat heart by GSK- $3 \beta$ inhibitor is attenuated: age-related changes in mitochondrial permeability transition pore modulation," American Journal of PhysiologyHeart and Circulatory Physiology, vol. 300, no. 3, pp. H922H930, 2011.

[67] E. N. Dedkova, L. K. Seidlmayer, and L. A. Blatter, "Mitochondria-mediated cardioprotection by trimetazidine in rabbit heart failure," Journal of Molecular and Cellular Cardiology, vol. 59, pp. 41-54, 2013.

[68] P. J. Oliveira, R. Seiça, P. M. Coxito et al., "Enhanced permeability transition explains the reduced calcium uptake in cardiac mitochondria from streptozotocin-induced diabetic rats," FEBS Letters, vol. 554, no. 3, pp. 511-514, 2003.

[69] D. J. Hausenloy, M. R. Duchen, and D. M. Yellon, "Inhibiting mitochondrial permeability transition pore opening at reperfusion protects against ischaemia-reperfusion injury," Cardiovascular Research, vol. 60, no. 3, pp. 617-625, 2003.

[70] M. Saotome, H. Katoh, Y. Yaguchi et al., "Transient opening of mitochondrial permeability transition pore by reactive oxygen species protects myocardium from ischemia-reperfusion injury," American Journal of Physiology-Heart and Circulatory Physiology, vol. 296, no. 4, pp. H1125-H1132, 2009.

[71] M. J. Berridge, P. Lipp, and M. D. Bootman, "The versatility and universality of calcium signalling," Nature Reviews Molecular Cell Biology, vol. 1, no. 1, pp. 11-21, 2000.

[72] A. B. Aurora, A. I. Mahmoud, X. Luo et al., "MicroRNA-214 protects the mouse heart from ischemic injury by controlling $\mathrm{Ca}^{2+}$ overload and cell death," The Journal of Clinical Investigation, vol. 122, no. 4, pp. 1222-1232, 2012.

[73] T. Liu, E. Takimoto, V. L. Dimaano et al., "Inhibiting mitochondrial $\mathrm{Na}^{+} / \mathrm{Ca}^{2+}$ exchange prevents sudden death in a Guinea pig model of heart failure," Circulation Research, vol. 115, no. 1, pp. 44-54, 2014.

[74] P. S. Brookes, Y. Yoon, J. L. Robotham, M. W. Anders, and S.S. Sheu, "Calcium, ATP, and ROS: a mitochondrial love-hate triangle," American Journal of Physiology-Cell Physiology, vol. 287, no. 4, pp. C817-C833, 2004.

[75] M. Aldakkak, A. K. S. Camara, J. S. Heisner, M. Yang, and D. F. Stowe, "Ranolazine reduces $\mathrm{Ca}^{2+}$ overload and oxidative stress and improves mitochondrial integrity to protect against ischemia reperfusion injury in isolated hearts," Pharmacological Research, vol. 64, no. 4, pp. 381-392, 2011.

[76] M. L. Riess, A. K. S. Camara, L. G. Kevin, J. An, and D. F. Stowe, "Reduced reactive $\mathrm{O}_{2}$ species formation and preserved mitochondrial NADH and $\left[\mathrm{Ca}^{2+}\right]$ levels during short-term 17 ${ }^{\circ} \mathrm{C}$ ischemia in intact hearts," Cardiovascular Research, vol. 61, no. 3, pp. 580-590, 2004.

[77] M. L. Riess, A. K. S. Camara, E. Novalija, Q. Chen, S. S. Rhodes, and D. F. Stowe, "Anesthetic preconditioning attenuates mitochondrial $\mathrm{Ca} 2+$ overload during ischemia in Guinea pig intact hearts: reversal by 5-hydroxydecanoic acid," Anesthesia and Analgesia, vol. 95, no. 6, pp. 1540-1546, 2002.

[78] R. Ferrari, P. Pedersini, M. Bongrazio et al., "Mitochondrial energy production and cation control in myocardial ischaemia and reperfusion," Basic Research in Cardiology, vol. 88, no. 5, pp. 495-512, 1993.

[79] D. De Stefani, A. Raffaello, E. Teardo, I. Szabó, and R. Rizzuto, "A forty-kilodalton protein of the inner membrane is the mitochondrial calcium uniporter," Nature, vol. 476, no. 7360 , pp. 336-340, 2011.

[80] D. De Stefani, M. Patron, and R. Rizzuto, "Structure and function of the mitochondrial calcium uniporter complex," Biochimica et Biophysica Acta (BBA)-Molecular Cell Research, vol. 1853, no. 9, pp. 2006-2011, 2015.

[81] T. M. Ye, S. Z. Zhang, and Q. Xia, "Role of mitochondrial calcium uniporter in myocardial hypoxia/reoxygenation induced injury," Chinese Journal of Applied Physiology, vol. 22, pp. 136140, 2006.

[82] M. Ahuja and S. Muallem, "The gatekeepers of mitochondrial calcium influx: MICU1 and MICU2," EMBO Reports, vol. 15, no. 3, pp. 205-206, 2014.

[83] K. J. Kamer and V. K. Mootha, "MICU1 and MICU2 play nonredundant roles in the regulation of the mitochondrial calcium uniporter," EMBO Reports, vol. 15, no. 3, pp. 299-307, 2014.

[84] K. Mallilankaraman, P. Doonan, C. Cárdenas et al., "MICU1 is an essential gatekeeper for mcu-mediated mitochondrial $\mathrm{Ca}^{2+}$ uptake that regulates cell survival," Cell, vol. 151, no. 3, pp. 630644, 2012.

[85] M. Patron, V. Checchetto, A. Raffaello et al., "MICU1 and MICU2 finely tune the mitochondrial $\mathrm{Ca}^{2+}$ uniporter by exerting opposite effects on MCU activity," Molecular Cell, vol. 53, no. 5, pp. 726-737, 2014.

[86] W. Wang, Q. Xie, X. Zhou et al., "Mitofusin-2 triggers mitochondria $\mathrm{Ca} 2+$ influx from the endoplasmic reticulum to induce apoptosis in hepatocellular carcinoma cells," Cancer Letters, vol. 358, no. 1, pp. 47-58, 2015.

[87] P. Michela, V. Velia, P. Aldo, and P. Ada, "Role of connexin 43 in cardiovascular diseases," European Journal of Pharmacology, vol. 768, pp. 71-76, 2015.

[88] A. Rodriguez-Sinovas, K. Boengler, A. Cabestrero et al., "Translocation of connexin 43 to the inner mitochondrial membrane of cardiomyocytes through the heat shock protein 90-dependent TOM pathway and its importance for cardioprotection," Circulation Research, vol. 99, no. 1, pp. 93-101, 2006.

[89] K.-G. Shyu, B.-W. Wang, W.-P. Cheng, and H.-M. Lo, "MicroRNA-208a increases myocardial endoglin expression and myocardial fibrosis in acute myocardial infarction," Canadian Journal of Cardiology, vol. 31, no. 5, pp. 679-690, 2015.

[90] R. Schulz, P. M. Görge, A. Görbe, P. Ferdinandy, P. D. Lampe, and L. Leybaert, "Connexin 43 is an emerging therapeutic target in ischemia/reperfusion injury, cardioprotection and neuroprotection," Pharmacology \& Therapeutics, vol. 153, pp. 90-106, 2015.

[91] N. Wang, E. De Vuyst, R. Ponsaerts et al., "Selective inhibition of Cx43 hemichannels by Gap19 and its impact on myocardial ischemia/reperfusion injury," Basic Research in Cardiology, vol. 108, article 309, 2013.

[92] G. Chen, J. Zhao, C. Liu, Y. Zhang, Y. Huo, and L. Zhou, "MG132 proteasome inhibitor upregulates the expression of connexin 
43 in rats with adriamycin-induced heart failure," Molecular Medicine Reports, vol. 12, no. 5, pp. 7595-7602, 2015.

[93] G. Heusch, J. Musiolik, N. Gedik, and A. Skyschally, "Mitochondrial STAT3 activation and cardioprotection by ischemic postconditioning in pigs with regional myocardial ischemia/reperfusion," Circulation Research, vol. 109, no. 11, pp. 1302-1308, 2011.

[94] K. Szczepanek, E. J. Lesnefsky, and A. C. Larner, "Multitasking: nuclear transcription factors with novel roles in the mitochondria," Trends in Cell Biology, vol. 22, no. 8, pp. 429437, 2012.

[95] F. A. Zouein, R. Altara, Q. Chen, E. J. Lesnefsky, M. Kurdi, and G. W. Booz, "Pivotal importance of STAT3 in protecting the heart from acute and chronic stress: new advancement and unresolved issues," Frontiers in Cardiovascular Medicine, vol. 2, article 36, 2015.

[96] K. Szczepanek, Q. Chen, A. C. Larner, and E. J. Lesnefsky, "Cytoprotection by the modulation of mitochondrial electron transport chain: the emerging role of mitochondrial STAT3," Mitochondrion, vol. 12, no. 2, pp. 180-189, 2012.

[97] J. Wegrzyn, R. Potla, Y.-J. Chwae et al., "Function of mitochondrial Stat3 in cellular respiration," Science, vol. 323, no. 5915, pp. 793-797, 2009.

[98] K. Szczepanek, Q. Chen, M. Derecka et al., "Mitochondrialtargeted signal transducer and activator of transcription 3 (STAT3) protects against ischemia-induced changes in the electron transport chain and the generation of reactive oxygen species," The Journal of Biological Chemistry, vol. 286, no. 34, pp. 29610-29620, 2011. 


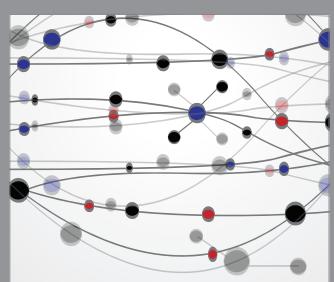

The Scientific World Journal
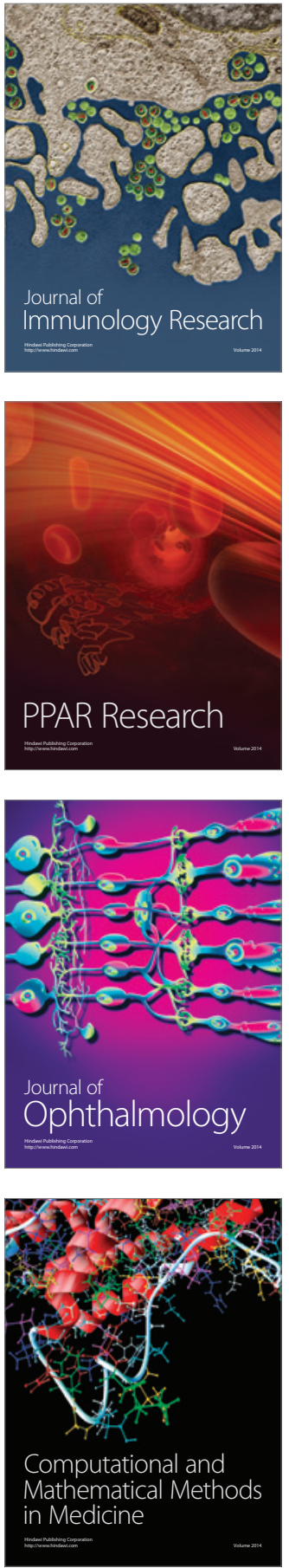

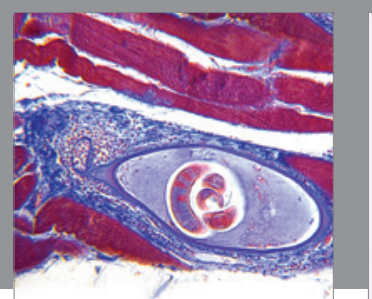

Gastroenterology Research and Practice

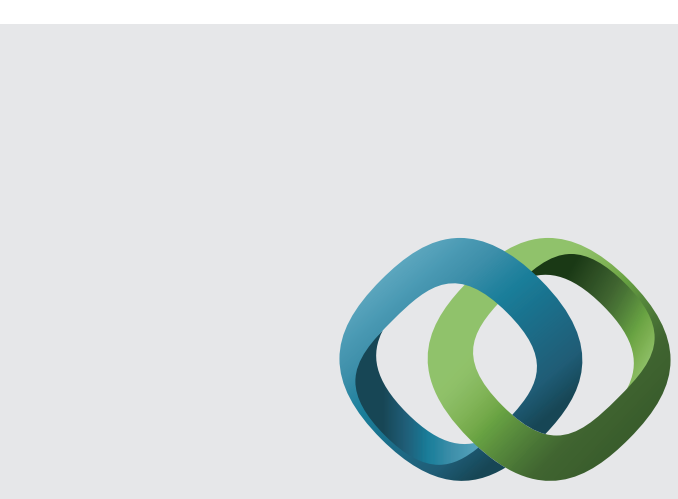

\section{Hindawi}

Submit your manuscripts at

http://www.hindawi.com
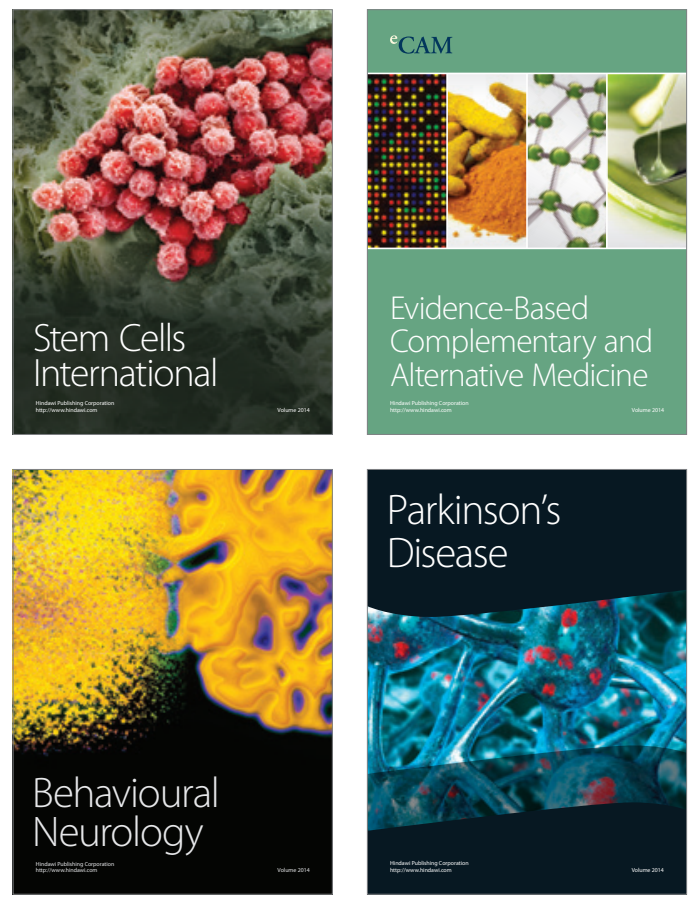
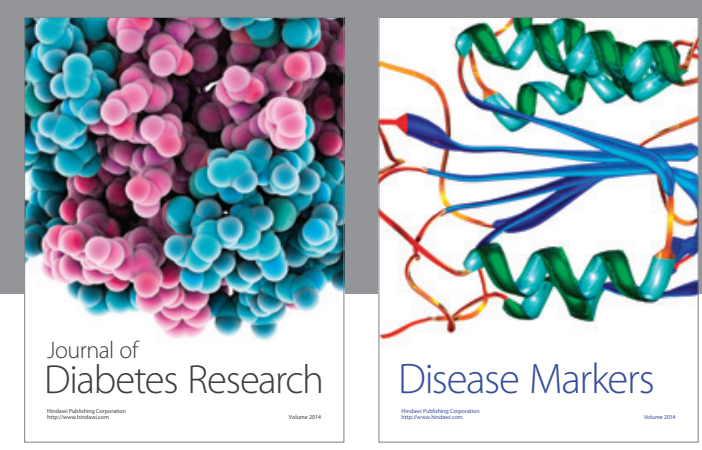

Disease Markers
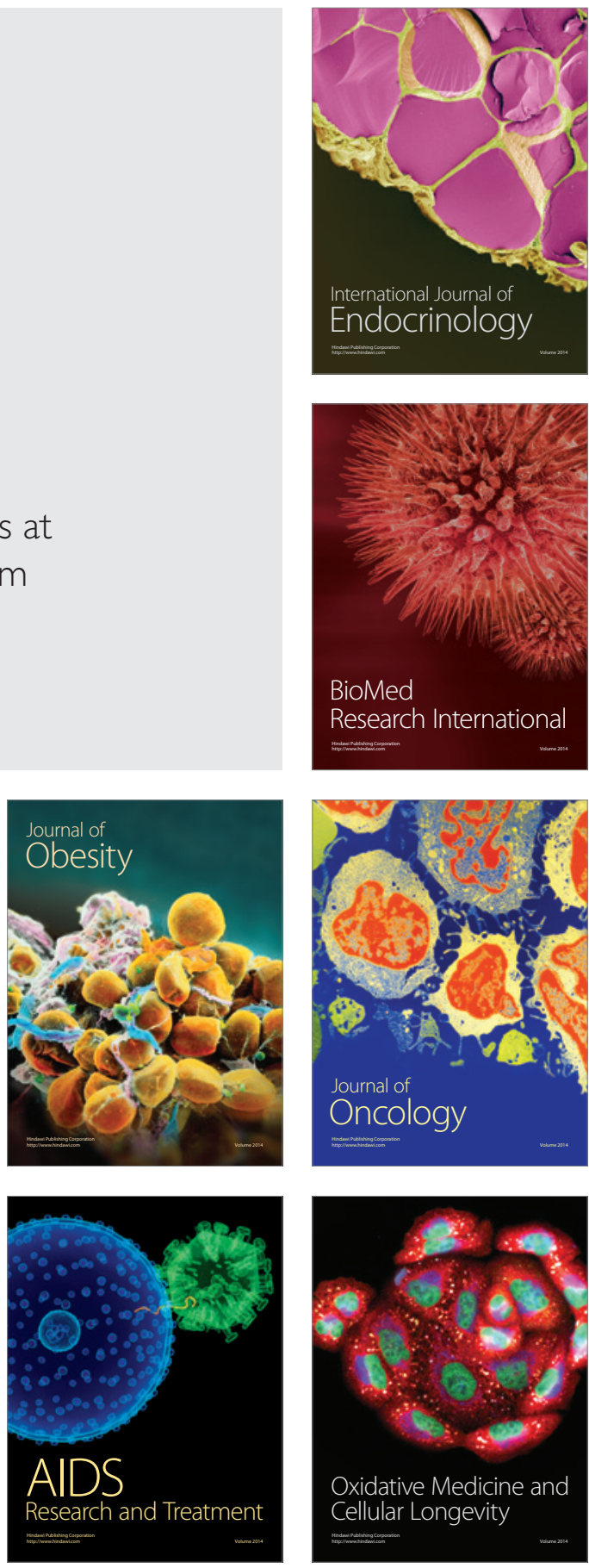